\title{
Meeting Recommended Levels of Physical Activity in Relation to Preventive Health Behavior and Health Status Among Adults
}

\author{
Peter D. Hart ${ }^{1,2}$, Gabriel Benavidez ${ }^{1,2}$, James Erickson ${ }^{1}$ \\ ${ }^{1}$ Health Promotion Program, Montana State University-Northern, Havre, MT; ${ }^{2}$ Kinesmetrics Lab, Montana State University-Northern, Havre, MT, USA
}

Objectives: The purpose of this study was to examine the relationship of meeting the recommended levels of physical activity (PA) with health status and preventive health behavior in adults.

Methods: A total of 5630 adults 18 years of age or older were included in this study. PA was assessed using a series of questions that categorized activities based on their metabolic equivalent values and then categorized individuals based on the reported frequency and duration of such activities. Participants reporting 150 minutes or more of moderate-intensity PA per week were considered to have met the PA guidelines. Multiple logistic regression was used to model the relationships between meeting PA guidelines and health status and preventive health behavior, while controlling for confounding variables.

Results: Overall, 53.9\% (95\% confidence interval [CI], 51.9 to 55.9\%) of adults reported meeting the recommended levels of PA. Among adults with good general health, $56.9 \%(95 \% \mathrm{Cl}, 54.7$ to $59.1 \%$ ) reported meeting the recommended levels of PA versus $43.1 \%$ (95\% Cl, 40.9 to $45.3 \%$ ) who did not. Adults who met the PA guidelines were significantly more likely not to report high cholesterol, diabetes, chronic obstructive pulmonary disease, arthritis, asthma, depression, or overweight. Furthermore, adults meeting the PA guidelines were significantly more likely to report having health insurance, consuming fruits daily, consuming vegetables daily, and not being a current cigarette smoker.

Conclusions: In this study, we found meeting the current guidelines for PA to have a protective relationship with both health status and health behavior in adults. Health promotion programs should focus on strategies that help individuals meet the current guidelines of at least 150 minutes per week of moderate-intensity PA.

Key words: Exercise, Quality of life, Health promotion, Health status, Population, Epidemiology

Received: August 26, 2016 Accepted: December 2, 2016

Corresponding author: Peter D. Hart, PhD

P.O. Box 7751 Havre, MT 59501-7751, USA

Tel: +1-406-265-3719, Fax: +1-406-265-4129

E-mail: peter.hart@msun.edu

This is an Open Access article distributed under the terms of the Creative Commons Attribution Non-Commercial License (http://creativecommons.org/licenses/by$\mathrm{nc} / 4.0 /$ ) which permits unrestricted non-commercial use, distribution, and reproduction in any medium, provided the original work is properly cited.

\section{INTRODUCTION}

As of 2014, seven of the 10 leading causes of death in the US were chronic diseases (heart disease, cancers, chronic lower respiratory diseases, cerebrovascular diseases, Alzheimer disease, diabetes mellitus, and influenza and pneumonia) [1]. Health risk behaviors are actions or inactions that increase an individual's risk for health problems such as chronic disease and consist of behaviors such as poor nutrition, tobacco use, 
alcohol abuse, and physical inactivity [2].

Physical activity (PA) is a health behavior for which strong evidence supports its protective effect against chronic diseases, as well as many risk factors for chronic disease, such as obesity, hypertension, dyslipidemia, and depression [3]. Current guidelines for PA recommend the accumulation of 150 minutes or more of moderate-intensity (or vigorous-intensity equivalent) aerobic physical activity each week [4]. The Healthy People 2020 objective regarding this guideline is to increase the percent of adults who meet this recommendation to $47.9 \%$ from the baseline of $43.5 \%$ [5].

Since the current PA guidelines were established, many researchers have attempted to study the relationship between meeting these guidelines and health outcomes. In one prospective study of osteoarthritic participants who wore accelerometers to objectively measure PA, quality-adjusted life-years were shown to be significantly greater among those meeting the PA guidelines than in their inactive counterparts [6]. A cross-sectional study of US adults, using self-reported PA, showed that adults residing in rural counties meeting recommended PA guidelines were more likely to report good healthrelated quality of life (HRQoL) than their counterparts who did not meet the PA guidelines [7]. Specifically, that study showed that rural adults accumulating at least 150 minutes of moderate-intensity PA were more likely to report good general health, physical health, mental health, and inactivity health. While some studies have shown the health benefits of meeting this specific PA guideline, less is known about its relationship with health status and preventive health behaviors in a state-based adult population.

Although a large percentage of adults meet the national PA objectives, with approximately $50 \%$ of adults getting $150 \mathrm{~min}$ utes or more of aerobic PA per week, it is unclear whether meeting these guidelines corresponds to improved health status or positive health behaviors [8]. Therefore, the purpose of this study was to examine the relationships between meeting the recommended levels of PA (150 minutes or more of moderate-intensity PA) and health status and preventive health behavior in adults.

\section{METHODS}

\section{Survey Design and Data}

Data for this study came from the Idaho Behavioral Risk Factor Surveillance System (BRFSS). The BRFSS is a state-based and territory-based survey administered through the Population Health Surveillance Branch of the Centers for Disease Control and Prevention (CDC) [9]. The purpose of the BRFSS is to collect state-based data on health risk and preventive behaviors associated with the leading causes of premature death and disability in the US. The health factors considered in the survey are alcohol and tobacco use, health care access, human immunodeficiency virus prevention, perceived health, health status, sleep quality, hypertension and cholesterol awareness, diet, arthritis burden, immunization, seatbelt use, and exercise behavior. BRFSS data collection relies on telephone-based surveys. As of 2011, both landlines and cellular phone lines were included. The 2013 survey year was used in this study because it was the most recent survey with appropriate questions for assessing adherence to the PA guidelines. A total of 5630 adults completed the 2013 survey and were included in the initial analysis.

\section{Measures}

PA was assessed based on responses to a series of survey questions regarding PA behavior during the previous 30 days [10]. The first step included estimating maximal oxygen consumption $\left(\mathrm{VO}_{2 \max }\right)$ and maximal metabolic equivalent $\left(\mathrm{MET}_{\max }\right)$ for each participant using age-predicted formulas for men $\left(\mathrm{VO}_{2 \max }=\left[60-0.55^{*}\right.\right.$ age in years $\left.]\right)$ and women $\left(\mathrm{VO}_{2 \max }=[48-\right.$ $0.37^{*}$ age in years]). Dividing the derived $\mathrm{VO}_{2 \max }$ values by 3.5 for each participant yielded a measure of $\mathrm{MET}_{\max }$. The second step included creating MET cutoffs for moderate-intensity and vigorous-intensity PA. This involved multiplying the MET $\mathrm{Max}_{\max }$ values from step 1 by 0.60 (60\%) to determine a vigorous-intensity threshold. Activities above this MET threshold were considered vigorous in intensity. Activities with MET values between three and the vigorous-intensity threshold were then considered moderate in intensity. The third step involved classifying each respondent's reported activity as vigorous, moderate, or neither. This step required matching the reported activities to activities contained in the 2011 Compendium of Physical Activities [11]. After the intensity coding, activities were then combined with the reported frequency (number of days) and duration (minutes that each activity lasted) for each activity to form a combined value of minutes of moderate PA (vigorous-intensity minutes were doubled to convert to a common moderate-intensity minutes scale). The final step was then to classify each participant as either meeting the PA guidelines or not meeting the guidelines. Those with at least 
150 minutes of moderate PA per week were considered to have met the PA guidelines. PA status was a dichotomous variable indicating meeting the PA guidelines.

Health status was assessed in this study based on responses to questions asking about specific health conditions. Measures of high blood pressure, high cholesterol, diabetes, chronic obstructive pulmonary disease (COPD), heart attack, stroke, arthritis, asthma, and depression were obtained from questions that asked the participant if a doctor, nurse, or health professional told them they had the condition [12]. Overweight status was derived from self-reported height and weight and the calculation of body mass index (BMI). Respondents with BMls greater than or equal to $25 \mathrm{~kg} / \mathrm{m}^{2}$ were considered overweight, and those with a BMI less than $25 \mathrm{~kg} / \mathrm{m}^{2}$ were considered not to be overweight [13]. As secondary indicators of health status, HRQoL was included and assessed by five different measures: general health, physical health, mental health, inactivity health, and healthy days [14]. General health was assessed using a single item asking participants to rate their own general health. Response options included "excellent," "very good,"' good," "fair," and "poor."Those reporting "excellent," "very good," or "good" general health were considered to have good general health. Those reporting "fair" or "poor" general health were considered to have poor general health. Physical and mental health measures were assessed using questions asking respondents to report the number of days (out of the previous 30 days) that their physical (or mental) health was not good. Those reporting 13 days or less were considered to have exhibited good physical (or mental) health [14]. Inactivity health was assessed using a question that specifically asked for the number of days (out of the previous 30 days) that poor physical or mental health kept them from their usual activities (self-care, work, or recreation). Those reporting 13 days or fewer were considered active in terms of health and therefore exhibited good inactivity health. Finally, a healthy days index was computed to represent the number of healthy (physical and mental) days out of the previous 30 . Those reporting 13 days or fewer were considered to have exhibited good HRQoL. All health status variables were dichotomized to indicate good health status.

Health behavior was assessed in this study based on responses to questions concerning either health risk activities or health protective activities [15]. Seatbelt use was assessed using a question asking participants how often they used seatbelts when they drove or rode in a car. Respondents answering "always" were considered to have engaged in preventive health behavior. Vegetable and fruit consumption was assessed similarly, using questions asking participants how often they ate vegetables (and fruit) in the past month. Participants responded in optional units of either per day, per week, or per month. Respondents averaging at least one serving of vegetable (and fruit) per day were considered to have engaged in preventive health behavior. Heavy alcohol consumption was assessed using an initial question asking participants how many days (out of the previous 30) per week or month they drank any alcoholic beverages, followed by a question asking how many drinks on average they consumed on those days. Participants reporting the consumption of two drinks or fewer (for males) or one drink or fewer (for females) per day were considered to have engaged in preventive health behavior. Binge-drinking behavior was assessed using a question asking participants how many times in the past 30 days they drank five or more (for males) or four or more (for females) drinks on a single occasion. Respondents reporting never having engaged in binge drinking in the past 30 days were considered to have engaged in preventive health behavior. Current smoking status was assessed using a question asking participants if they smoke cigarettes every day, some days, formerly, or not at all. Respondents reporting "formerly" or "not at all" to that question were considered to have engaged in preventive health behavior. The final health behavior, having health insurance, was assessed using a question asking participants if they had any kind of health care coverage. Respondents reporting "yes" to that question were considered to have engaged in preventive health behavior. All health behavior variables were dichotomized to indicate preventive health behavior.

Control variables were used in this study both to describe the sample and to control for their confounding effects. Age (18-24, 25-34, 35-44, 45-54, 55-64, or 65+ years), sex (male or female), race (white, black, Hispanic, multicultural, or other), education (less than high school, high school graduate, some college, or college graduate), income (<15 000; 15 000-24 999; 25 000-34 999; 35 000-49 999; or $\geq 50000$ USD), and marital status (married or not married) were used to describe the sample.

\section{Statistical Analysis}

Prevalence estimates (\%) and 95\% confidence intervals (Cls) were computed for PA status across demographic categories and HRQoL measures with associated Rao-Scott chi-square statistics [16]. Odds ratios (ORs) and 95\% Cls were computed 
from logistic and multiple logistic regression using the health measures (HRQoL, health status, preventive health behavior) as dependent variables and PA status as the primary independent variable [17]. ORs were computed across BMI categories using logistic regression and the Cochran-Armitage test of trend [18]. The fully adjusted logistic regression model included age, sex, race, education, income, and marital status. SAS version 9.4 (SAS Institute Inc., Cary, NC, USA) survey proce-

Table 1. Prevalence of meeting and not meeting the recommended levels of physical activity (PA) by demographic category in adults in Idaho, 2013

\begin{tabular}{|c|c|c|c|}
\hline & \multicolumn{2}{|c|}{ Meet PA guidelines ${ }^{1}$} & \multirow{2}{*}{$\begin{array}{c}p- \\
\text { value }^{2}\end{array}$} \\
\hline & Yes & No & \\
\hline Overall $(n=5078)$ & $53.9(51.9,55.9)$ & $46.1(44.1,48.2)$ & $<0.001$ \\
\hline $\operatorname{Sex}(n=5078)$ & & & 0.72 \\
\hline Male & $53.5(50.4,56.6)$ & $46.5(43.4,49.6)$ & \\
\hline Female & $54.2(51.6,56.9)$ & $45.8(43.1,48.4)$ & \\
\hline Age $(y, n=5078)$ & & & 0.003 \\
\hline $18-24$ & $55.3(48.1,62.5)$ & $44.7(37.5,51.9)$ & \\
\hline $25-34$ & $49.5(44.3,54.8)$ & $50.5(45.2,55.7)$ & \\
\hline $35-44$ & $52.8(47.5,58.2)$ & $47.2(41.9,52.5)$ & \\
\hline $45-54$ & $48.2(43.5,52.9)$ & $51.8(47.1,56.5)$ & \\
\hline $55-64$ & $56.0(51.7,60.3)$ & $44.0(39.8,48.3)$ & \\
\hline$\geq 65$ & $61.3(58.1,64.5)$ & $38.7(35.6,41.9)$ & \\
\hline Race/ethnicity ( $n=5007)$ & & & 0.01 \\
\hline White & $54.7(52.7,56.8)$ & $45.3(43.2,47.4)$ & \\
\hline Black & $58.7(22.7,94.8)$ & $41.3(5.3,77.4)$ & \\
\hline Hispanic & $42.0(33.3,50.6)$ & $58.0(49.4,66.7)$ & \\
\hline Multiracial & $61.0(44.9,77.1)$ & $39.0(22.9,55.2)$ & \\
\hline Other & $67.5(55.2,79.8)$ & $32.5(20.2,44.9)$ & \\
\hline Education $(n=5071)$ & & & $<0.001$ \\
\hline$<$ High school & $39.8(32.4,47.2)$ & $60.2(52.8,67.6)$ & \\
\hline High school graduate & $49.1(45.2,52.9)$ & $50.9(47.1,54.8)$ & \\
\hline Some college & $55.5(52.2,58.8)$ & $44.5(41.2,47.8)$ & \\
\hline College graduate & $64.4(61.1,67.6)$ & $35.6(32.4,38.9)$ & \\
\hline Marital status $(n=5052)$ & & & 0.51 \\
\hline Married & $54.5(52.0,56.9)$ & $45.6(43.1,48.0)$ & \\
\hline Not married & $53.0(49.6,56.5)$ & $47.0(43.5,50.4)$ & \\
\hline Income (USD, $n=4540$ ) & & & $<0.001$ \\
\hline$<15000$ & $42.2(35.5,48.9)$ & $57.8(51.1,64.5)$ & \\
\hline $15000-24999$ & $47.6(42.5,52.8)$ & $52.4(47.2,57.5)$ & \\
\hline 25 000-34 999 & $49.0(43.5,54.5)$ & $51.0(45.5,56.6)$ & \\
\hline $35000-49999$ & $56.0(50.8,61.2)$ & $44.0(38.8,49.2)$ & \\
\hline$\geq 50000$ & $60.3(57.0,63.5)$ & $39.7(36.5,43.0)$ & \\
\hline
\end{tabular}

Values are presented as odds ratio ( $95 \%$ confidence interval).

${ }^{1}$ The cutoff for meeting the PA guidelines was 150 minutes or more of moderate-intensity PA (or the vigorous equivalent thereof).

${ }^{2} p$-values were determined using the Rao-Scott chi-square statistic. dures and SPSS version 24 (IBM Corp., Armonk, NY, USA) complex samples were used for all analyses $[19,20]$.

\section{RESULTS}

Of the 5630 participants included in the initial analysis, 5078 had complete PA data, with $53.9 \%(95 \% \mathrm{Cl}, 51.9$ to $55.9 \%)$ meeting the PA guidelines and $46.1 \%(95 \% \mathrm{Cl}, 44.1$ to $48.2 \%)$ not meeting the guidelines. No differences according to sex or marital status were seen in the prevalence of meeting the PA guidelines (Table 1). However, significant differences in prevalence across groups of age, race, education, and income were seen $(p<0.05$ for all). HRQoL was significantly related to meeting the PA guidelines (Table 2), with a greater prevalence of meeting the guidelines found among those with good $\mathrm{HRQOL}$ than among those with poor HRQoL on measures of general health $(56.9 \%$ vs. $35.9 \%, p<0.001)$, physical health $(55.8 \%$ vs. $38.3 \%, p<0.001)$, mental health ( $55.9 \%$ vs. $42.1 \%, p<0.001)$, inactivity health $(51.8 \%$ vs. $34.4 \%, p<0.001)$, and healthy days (57.0\% vs. $43.3 \%, p<0.001)$.

Table 3 displays the results of the logistic regression analyses with unadjusted, age-adjusted, and fully adjusted (age,

Table 2. Prevalence of meeting and not meeting the recommended levels of PA by HRQoL measures in adults in Idaho, 2013

\begin{tabular}{lccc}
\hline & \multicolumn{2}{c}{ Meet PA guidelines ${ }^{1}$} & \\
\cline { 2 - 3 } & \multicolumn{1}{c}{ Yes } & No & \\
\hline General health $(n=5052)$ & & & $<0.001$ \\
Good & $56.9(54.7,59.1)$ & $43.1(40.9,45.3)$ & \\
Poor & $35.9(30.9,40.8)$ & $64.1(59.2,69.1)$ & \\
Physical health $(n=4971)$ & & & $<0.001$ \\
Good & $55.8(53.7,58.0)$ & $44.2(42.0,46.4)$ & \\
Poor & $38.3(33.0,43.6)$ & $61.7(56.4,67.0)$ & \\
Mental health $(n=4993)$ & & & $<0.001$ \\
Good & $55.9(53.8,58.1)$ & $44.1(41.9,46.2)$ & \\
Poor & $42.1(36.0,48.2)$ & $57.9(51.8,64.0)$ & \\
Inactivity health $(n=2715)$ & & & $<0.001$ \\
Good & $51.8(48.7,54.8)$ & $48.2(45.2,51.3)$ & \\
Poor & $34.4(28.2,40.6)$ & $65.6(59.4,71.8)$ & \\
Healthy days $(n=4908)$ & & & $<0.001$ \\
Good & $57.0(54.7,59.3)$ & $43.0(40.7,45.3)$ & \\
Poor & $43.3(38.9,47.8)$ & $56.7(52.2,61.1)$ & \\
\hline
\end{tabular}

Values are presented as odds ratio ( $95 \%$ confidence interval).

PA, physical activity; HROoL, health-related quality of life.

${ }^{1}$ The cutoff for meeting the PA guidelines was 150 minutes or more of moderate-intensity PA (or the vigorous equivalent thereof).

${ }^{2} p$-values were determined using the Rao-Scott chi-square statistic. 
sex, race, education, income, and marital status) ORs reported. Adults who met the PA guidelines were significantly more likely to report good HRQoL across all measures (OR range, 1.59 to $1.99, p<0.001$ for all) after full adjustment. In fully adjusted models of health status measures, those meeting the PA guidelines were significantly more likely not to report high cholesterol (OR, 1.39; $p=0.002)$, diabetes (OR, 1.50, $p=0.01)$, COPD (OR, 1.72, $p=0.004)$, arthritis (OR, 1.26, $p<0.05)$, asthma $(\mathrm{OR}, 1.34, p<0.05)$, depression (OR, 1.54, $p<0.001)$, or overweight (OR, 1.48, $p<0.001)$. ORs for high blood pressure and heart attack were not significant in either the adjusted or unadjusted models, whereas stroke was significant in both the unadjusted $(\mathrm{OR}, 1.97, p=0.004)$ and age-adjusted $(\mathrm{OR}, 2.33$, $p<0.001)$ models but not the fully adjusted model. In the fully adjusted model of preventive health behavior measures, adults meeting the PA guidelines were significantly more likely to report having health insurance $(\mathrm{OR}, 1.39, p=0.01)$, consuming fruits $(\mathrm{OR}, 1.69, p<0.001)$, consuming vegetables $(\mathrm{OR}, 1.67$, $p<0.001)$, and not being a current cigarette smoker (OR, 1.97, $p<0.001)$. Heavy alcohol consumption and binge drinking were not significant in either the unadjusted or adjusted models. However, seatbelt use was significant in the unadjusted model (OR, 1.24, $p<0.05)$, but not significant in either of the adjusted models.

Figure 1 displays the ORs for meeting the PA guidelines across the BMI categories of normal-weight, overweight, and

Table 3. Odds of good HRQoL, good health status, and preventive health behavior among adults meeting the recommended levels of PA in Idaho, 2013

\begin{tabular}{|c|c|c|c|}
\hline \multirow{2}{*}{ Health indicator } & \multicolumn{3}{|c|}{ Meets PA guidelines } \\
\hline & Unadjusted & Age-adjusted & Fully adjusted' \\
\hline \multicolumn{4}{|l|}{ Good HROoL } \\
\hline Physical health & $2.04(1.60,2.59)$ & $2.20(1.71,2.83)$ & $1.75(1.33,2.31)$ \\
\hline Mental health & $1.75(1.34,2.27)$ & $1.76(1.35,2.31)$ & $1.59(1.19,2.12)$ \\
\hline \multicolumn{4}{|l|}{ Good health status } \\
\hline High blood pressure & $0.99(0.84,1.18)$ & $0.85(0.70,1.03)$ & $0.88(0.71,1.08)$ \\
\hline High cholesterol & $1.35(1.13,1.62)$ & $1.46(1.20,1.78)$ & $1.39(1.13,1.72)$ \\
\hline Diabetes & $1.44(1.11,1.87)$ & $1.67(1.27,2.20)$ & $1.50(1.10,2.04)$ \\
\hline Arthritis & $1.12(0.94,1.33)$ & $1.33(1.10,1.62)$ & $1.26(1.02,1.55)$ \\
\hline Asthma & $1.46(1.15,1.86)$ & $1.46(1.15,1.86)$ & $1.34(1.03,1.73)$ \\
\hline Depression & $1.60(1.32,1.95)$ & $1.60(1.31,1.95)$ & $1.54(1.24,1.91)$ \\
\hline Overweight & $1.46(1.22,1.74)$ & $1.50(1.25,1.80)$ & $1.48(1.21,1.80)$ \\
\hline \multicolumn{4}{|l|}{ Preventive health behavior } \\
\hline Always uses seatbelt & $1.24(1.01,1.53)$ & $1.20(0.98,1.48)$ & $1.16(0.93,1.45)$ \\
\hline Consumes vegetables daily & $1.90(1.54,2.36)$ & $1.85(1.49,2.30)$ & $1.67(1.31,2.13)$ \\
\hline Consumes fruits daily & $1.91(1.61,2.27)$ & $1.85(1.55,2.20)$ & $1.69(1.41,2.04)$ \\
\hline Non-heavy alcohol drinker & $0.99(0.70,1.39)$ & $0.99(0.70,1.39)$ & $1.07(0.75,1.51)$ \\
\hline Does not binge drink & $0.95(0.74,1.22)$ & $0.88(0.68,1.14)$ & $0.94(0.72,1.24)$ \\
\hline
\end{tabular}

Values are presented as odds ratio ( $95 \%$ confidence interval).

The comparison group was those who did not meet the recommended levels of PA.

HRQoL, health-related quality of life; PA, physical activity; COPD, chronic obstructive pulmonary disease.

${ }^{1}$ Fully adjusted logistic regression models controlled for age, sex, race, education, income, and marital status.

${ }^{2}$ Due to a quasi-complete separation of data points, race was dichotomized to white vs. other in this model only. All health status variables (except overweight) were assessed from answers to questions asking participants if a health professional ever told them that they had the condition in question. 




Figure 1. Odds ratios (ORs) for meeting the recommended levels of physical activity across body mass index categories in adults in Idaho, 2013. The obese category is the reference group for the ORs. 'Represents a significant OR compared to the reference group.

obese adults. Overall, normal weight $(\mathrm{OR}, 1.65 ; 95 \% \mathrm{Cl}, 1.34$ to 2.04) and overweight (OR, 1.26; $95 \% \mathrm{Cl}, 1.03$ to 1.55$)$ adults were significantly more likely to report meeting the PA guidelines than obese adults. The overall ORs showed a significant linear trend $(p<0.001)$. When stratified by age group, the youngest age group (18 to 44 years) showed neither a signifcant increase in odds for either BMI category nor a significant trend in ORs. However, the middle-age group (45 to 64 years) showed significantly greater odds of reporting meeting the PA guidelines in normal-weight $(\mathrm{OR}, 2.50 ; 95 \% \mathrm{Cl}, 1.79$ to 3.50$)$ and overweight adults (OR, 1.60; $95 \% \mathrm{Cl}, 1.16$ to 2.20 ) than in obese adults. As well, in the older-age group ( $\geq 65$ years), normal-weight (OR, 2.07; $95 \% \mathrm{Cl}, 1.50$ to 2.94 ) and overweight $(\mathrm{OR}, 2.20 ; 95 \% \mathrm{Cl}, 1.56$ to 3.10$)$ adults were significantly more likely to report meeting the PA guidelines than obese adults. Both the middle-age and older-age groups showed a significant linear trend in ORs $(p<0.001)$.

\section{DISCUSSION}

The purpose of this study was to examine the relationship between meeting the recommended levels of PA and health status and preventive health behavior in adults. The results of this study clearly showed that meeting the PA guidelines was related to good health status in adults. After considering age, sex, race, education, income, and marital status, adults who met these guidelines were more likely to report positive health concerning cholesterol, diabetes, COPD, arthritis, asthma, depression, and overweight. These findings are consistent with those of other studies. For example, a prospective study examined the relationship between accelerometer-determined PA and high-density lipoprotein (HDL) cholesterol [21]. After adjusting for potential confounders, results from this study clearly showed that participants who engaged in greater amounts of PA (step/d) had significantly higher HDL levels. Similarly, a meta-analysis of nine prospective studies examined the combined and independent effects of PA and BMI on the development of type 2 diabetes [22]. The results of this analysis showed that the combined effects of being normal weight and having high PA were significantly related with a decreased risk of developing type 2 diabetes. Furthermore, participants who were obese and had low levels of PA were over seven times more likely to develop type 2 diabetes.

To our surprise, we found that meeting the PA guidelines was not related to positive health status concerning blood pressure, heart disease, or stroke. This paradoxical finding may in part be due to the relatively small number of participants reporting a heart attack or stroke. The effect sizes on the ORs were large (>1.31) across all models for both heart attack and stroke indicators; however, the small sample size limited the power of these models [23].

When considering HRQoL as a secondary indicator of health status, however, meeting PA guidelines was related to all such measures (general health, physical health, mental health, inactivity health, and healthy days). These findings are also consistent with contemporary research outcomes. For instance, a large study of over 5000 patients enrolled in a dialysis study examined the prospective effects of PA (measured as five different levels from never to very active) on HRQoL (measured with the Kidney Disease Quality of Life Scale) [24]. Using linear regression, researchers reported a significant positive relationship between PA and HRQoL in that study population.

The results of this study also showed that meeting the PA guidelines was positively related to preventive health behavior in adults. After considering the confounding variables, meeting the PA guidelines was related to preventive behavior concerning vegetable consumption, fruit consumption, cigarette smoking, and health insurance. These results are also consistent with currently published findings. For example, a large population-level study of US adults examined the relationship between accelerometer-determined PA and healthy diet behavior. That study found that moderate-to-vigorous PA was significantly and directly related to the consumption of fruits (apples, grapes, strawberries, and oranges) and vegeta- 
bles (greens, carrots, and peppers) [25]. Our study findings, however, showed that meeting the PA guidelines was not related to preventive health behavior concerning seatbelt use, heavy alcohol consumption, or binge drinking.

This study has limitations associated with its findings. One limitation of this study is its cross-sectional research design. Cross-sectional designs are limited to correlational inferences, as opposed to cause-and-effect findings that can be identified using experimental designs. Regardless of this limitation, the findings of this study are consistent with those of other crosssectional studies. For example, a large nationwide study of college students found that meeting the PA guidelines was related to fruit and vegetable consumption, perceived general health, healthy BMl, and not smoking cigarettes [26].

Another limitation of this study is that the data were collected via telephone. The limitation to this aspect of the design is that certain segments of the population may be less likely to have access to a telephone. These subpopulations may also be less likely to be physically active, more likely to be of poor health status, and less likely to engage in preventive health behavior. However, there is reason to speculate that including more respondents from such segments would only increase the strength of the relationships we observed. A final limitation of this study is the use of self-reported assessments of PA, health status, and health behavior. Although these limitations are valid, it stands to reason that the use of five different measures of HRQoL, 10 measures of health status, and seven measures of health behavior would provide a certain degree of confidence that the findings in this study are robust with regard to such limitations.

This study has several strengths. One strength of this study is that it was based on a large statewide survey that was representative of all adults. Many studies examining the relationships between PA and other health measures have not utilized large representative samples, and this fact strengthens our generalizations. A second strength of this study is that it was capable of quantifying the amounts of PA that participants engaged in to determine if they met the current guidelines of 150 minutes or more of moderate-intensity PA per week. By doing so, we were able to examine the relationship of PA with health status and health behavior more specifically in terms of meeting the current PA guidelines.

In summary, the present study found that meeting the current recommended guidelines for PA had a positive relationship with both good health status and preventive health be- havior in adults. Health promotion programs should focus on strategies that help adults meet the current guidelines of at least 150 minutes per week of moderate-intensity (or vigorous-intensity equivalent) PA.

\section{CONFLICT OF INTEREST}

The authors have no conflicts of interest associated with the material presented in this paper.

\section{ORCID}

Peter D. Hart https://orcid.org/0000-0002-4947-9310

Gabriel Benavidez https://orcid.org/0000-0003-2268-6229

James Erickson https://orcid.org/0000-0002-4745-0610

\section{REFERENCES}

1. National Center for Health Statistics. Health, United States, 2015 with special feature on racial and ethnic health disparities [cited 2016 Dec 27]. Available from: https://www.cdc.gov/ nchs/data/hus/hus15.pdf.

2. Centers for Disease Control and Prevention. Chronic disease prevention and health promotion: chronic disease overview [cited 2016 Dec 27]. Available from: https://www.cdc.gov/ chronicdisease/overview/index.htm.

3. Garber CE, Blissmer B, Deschenes MR, Franklin BA, Lamonte MJ, Lee IM, et al. American College of Sports Medicine position stand. Quantity and quality of exercise for developing and maintaining cardiorespiratory, musculoskeletal, and neuromotor fitness in apparently healthy adults: guidance for prescribing exercise. Med Sci Sports Exerc 2011;43(7):1334-1359.

4. US Department of Health and Human Services. 2008 Physical activity guidelines for americans fact sheet for health professionals on physical activity guidelines for adults [cited 2016 Dec 27]. Available from: https://www.cdc.gov/physicalactivity/downloads/pa_fact_sheet_adults.pdf.

5. US Department of Health and Human Services. Developing healthy people 2020 [cited 2016 Dec 27]. Available from: http://www.healthypeople.gov/2010/hp2020/Comments/ default.asp.

6. Sun K, Song J, Manheim LM, Chang RW, Kwoh KC, Semanik PA, et al. Relationship of meeting physical activity guidelines with quality-adjusted life-years. Semin Arthritis Rheum 2014;44 (3):264-270. 
7. Hart PD. Meeting recommended levels of physical activity and health-related quality of life in rural adults. J Lifestyle Med 2016;6(1):1-6.

8. National Center for Chronic Disease Prevention and Health Promotion. Behavioral Risk Factor Surveillance System: prevalence data \& data analysis tools [cited 2017 Jan 5]. Available from: https://www.cdc.gov/brfss/data_tools.htm.

9. Centers for Disease Control and Prevention. Behavioral Risk Factor Surveillance System: overview: BRFSS 2013; 2014 [cited 2017 Jan 5]. Available from: https://www.cdc.gov/brfss/annual_data/2013/pdf/overview_2013.pdf.

10. Centers for Disease Control and Prevention. A data users guide to the BRFSS physical activity questions: how to assess the 2008 physical activity guidelines for Americans [cited 2016 Dec 27]. Available from: http://www.cdc.gov/brfss/pdf/PA\%20 RotatingCore_BRFSSGuide_508Comp_07252013FINAL.pdf.

11. Ainsworth BE, Haskell WL, Herrmann SD, Meckes N, Bassett DR Jr, Tudor-Locke C, et al. 2011 Compendium of Physical Activities: a second update of codes and MET values. Med Sci Sports Exerc 2011;43(8):1575-1581.

12. Centers for Disease Control and Prevention. Calculated variables in the data file of the 2013 behavioral Risk Factor Surveillance System; 2014 [cited 2017 Jan 5]. Available from: https://www.cdc.gov/brfss/annual_data/2013/pdf/2013_calculated_variables_version15.pdf.

13. World Health Organization. BMI classification [cited 2016 Aug 26]. Available from: http://apps.who.int/bmi/index.jsp? introPage $=$ intro_3.html.

14. Moriarty DG, Zack MM, Kobau R. The Centers for Disease Control and Prevention's Healthy Days Measures: population tracking of perceived physical and mental health over time. Health Qual Life Outcomes 2003;1:37.

15. Centers for Disease Control and Prevention. Behavioral Risk Factor Surveillance System: 2013 codebook report: land-line and cell-phone data; 2014 [cited 2017 Jan 5]. Available from: https://www.cdc.gov/brfss/annual_data/2013/pdf/codebook13_llcp.pdf.

16. Agresti A, Kateri M. Categorical data analysis. Berlin: Springer;
2011, p. 163-195.

17. Tabachnick BG, Fidell LS. Using multivariate statistics. 5th ed. Boston: Allyn \& Bacon; 2007, p. 439-484.

18. Stokes ME, Davis CS, Koch GG. Categorical data analysis using SAS. Cary: SAS institute; 2012, p. 189-258.

19. SAS Institute. Base SAS 9.4 procedures guide: statistical procedures; 2014 [cited 2016 Dec 27]. Available from: https://support.sas.com/documentation/cdl/en/procstat/67528/PDF/ default/procstat.pdf.

20. IBM Corporation. IBM SPSS complex samples: correctly compute complex samples statistics [cited 2017 Jan 5]. Available from: http://www-01.ibm.com/common/ssi/cgi-bin/ssialias?h tmlfid $=$ YTD03116USEN\&appname $=$ skmwww.

21. Matsuzawa R, Matsunaga A, Kutsuna T, Ishii A, Abe Y, Yoneki K, et al. Association of habitual physical activity measured by an accelerometer with high-density lipoprotein cholesterol levels in maintenance hemodialysis patients. ScientificWorldJournal 2013;2013:780783.

22. Cloostermans L, Wendel-Vos W, Doornbos G, Howard B, Craig $\mathrm{CL}$, Kivimäki $\mathrm{M}$, et al. Independent and combined effects of physical activity and body mass index on the development of type 2 diabetes: a meta-analysis of 9 prospective cohort studies. Int J Behav Nutr Phys Act 2015;12:147.

23. Rosner B. Fundamentals of biostatistics. 7th ed. Boston: Cengage Learning; 2011, p. 628-657.

24. Lopes AA, Lantz B, Morgenstern H, Wang M, Bieber BA, Gillespie BW, et al. Associations of self-reported physical activity types and levels with quality of life, depression symptoms, and mortality in hemodialysis patients: the DOPPS. Clin J Am Soc Nephrol 2014;9(10):1702-1712.

25. Loprinzi PD. Association between accelerometer-determined physical activity and flavonoid-rich fruit and vegetable consumption among a national sample of US adults. Pre Med Rep 2016;3:58-61.

26. Dinger MK, Brittain DR, Hutchinson SR. Associations between physical activity and health-related factors in a national sample of college students. J Am Coll Health 2014;62(1):67-74. 